Antimicrobial activity was not activated by $\mathrm{N}$-substitution. When the structure of $\mathrm{AB}$ ring in steroids was 3,5-diene or 5-ene, the effect of substituents was to keep or weaken the activity of original compounds, and magnitudes of cativity were in the order of $\left.\mathrm{CH}_{2} \mathrm{CH}_{2} \mathrm{CN}\right\rangle\left(\mathrm{CH}_{2^{-}}\right.$ $\left.\mathrm{CH}_{2} \mathrm{OH}\right)_{2}>\left(\mathrm{CH}_{2} \mathrm{CH}_{2} \mathrm{Cl}\right)_{2} \gg\left(\mathrm{CH}_{2}\right)_{3} \mathrm{NH}_{2}$. The compounds derived from inactive XVII were all inactive, therefore, in 5 -en-3 $\beta$-ol no activation effect was observed.

Substitution of aminopropyl group to $17 \beta$-aminosteroids brought VII, XIV, and XIX marked anti-inflammatory action.

Acknowledgement The author is grateful to Prof. Y. Urushibara of Sophia University and Prof. K. Nakanishi of Chemical Department Tohoku University for their encouragements, to Drs. I. Chuman and $\mathrm{H}$. Ando of Teikoku Hormone Mfg. Co., Ltd. for their interest and support. Thanks are also due to Drs. R. Suzuki and M. Tarutani for the examination of anti-inflammatory action.

\author{
Research Laboratory, \\ Teikoku Hormone. Mfg. Co., Ltd., \\ Shimosakunobe, Kawasaki
}

Received December 13, 1968
YoSHIRO NAGAI

\title{
The Relationship between the Hydroxylated Position of Aromatic Compounds by Monooxygenase and Various Electronic Reactivity Indices
}

It has been recognized that a large number of both normally occurring and foreign aromatic compounds are hydroxylated by monooxygenase in the animal body to form phenolic compounds. However, little information is available concerning the biochemical mechanisms involved, and the extent of hydroxylation and orientation of substituting group have been the subjects of a vast number of studies with intact animals.

At this time, if a common theory by which hydroxylation reaction of aromatic compounds by monooxygenase may be illustrated uniformly at an electronic level were to be presented, it would be very important to clarify the reaction of monooxygenase and also useful to predict the structure of hydroxylated compounds of various drugs. In this work reported here, the $\pi$-electron densities and free valence numbers in the case of ground and first electronic excited state were obtained for some of aromatic substances by a molecular orbital method, simple LCAO MO method for $\pi$-electrons.

The parameters of coulomb and resonance integrals for substituent groups are the same as the values cited in previous papers. $\left.{ }^{1,2}\right)$

These results are shown in Fig. 1. In this respect, the $\pi$-electron density is a measure of nucleophilic and electrophilic reactions and the free valence number is a measure of radical reaction.

1) S. Hata, M. Yamakawa, S. Tomioka and H. Sano, Chem. Pharm. Bull., (Tokyo), 13, 96 (1965).

2) S. Hata, K. Mizuno and S. Tomioka, Yakuzaigaku, 27, 130 (1967). 


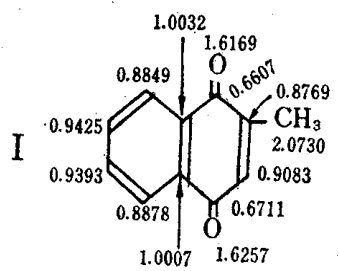

qr

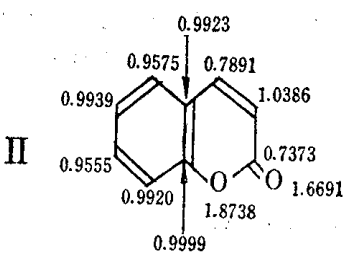

$q \mathrm{r}$

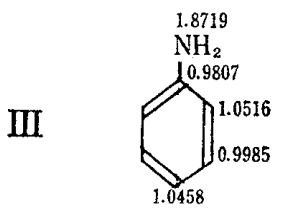

$q \mathbf{r}$<smiles>Cc1ccncc1N</smiles>

$q r$

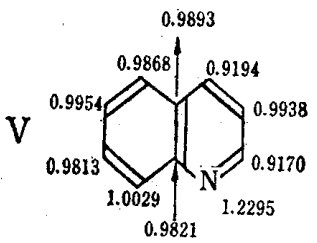

$q \mathbf{r}$

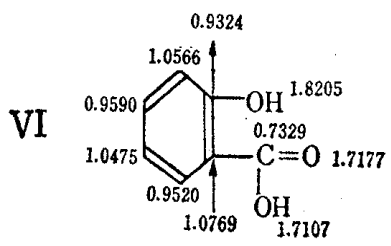

$\mathrm{qr}$

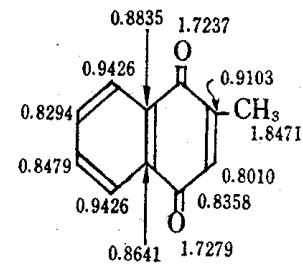

$\mathrm{q} \stackrel{*}{\mathrm{r}}$

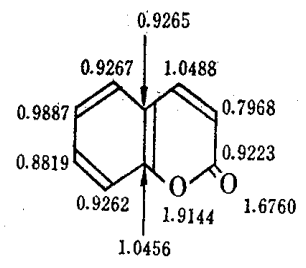

$q \stackrel{*}{r}$

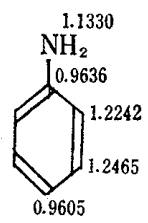

$q \stackrel{*}{r}$

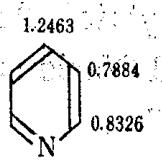

1.5106

$q \stackrel{*}{r}$

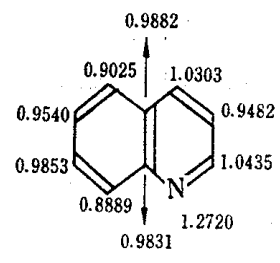

$q \stackrel{*}{r}$

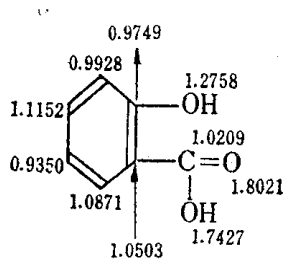

$\mathrm{q} \stackrel{*}{\mathrm{r}}$

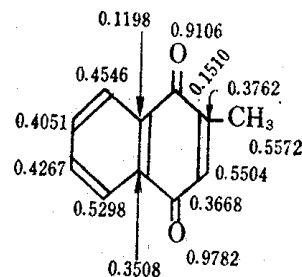

Fr

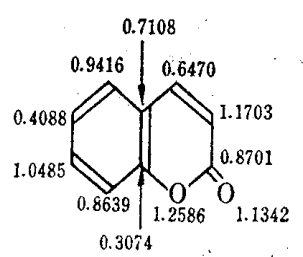

$\mathrm{Fr}$

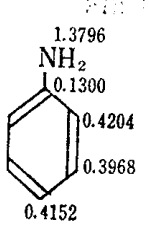

Fr

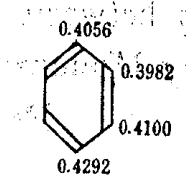

Fr

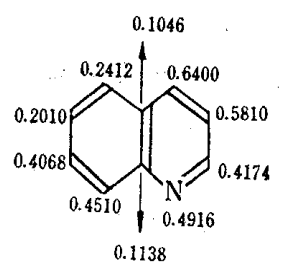

Fr

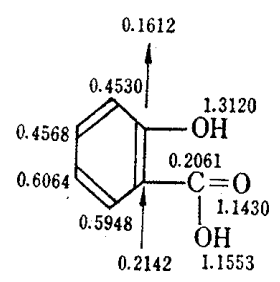

Fr

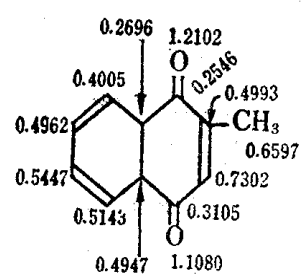

$\mathrm{Fr}_{\mathrm{r}}^{*}$

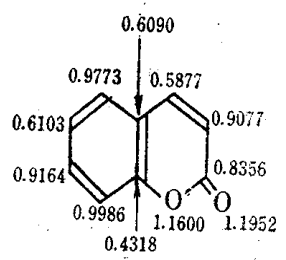

$\mathrm{Fr}^{*}$<smiles>Nc1ccccc1</smiles>

Fr

$\mathrm{Fr}_{\mathrm{r}}^{*}$

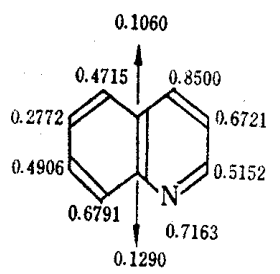

$\mathrm{Fr}_{\mathrm{r}}^{*}$

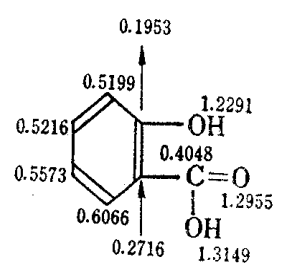

$\mathrm{Fr}_{\mathrm{r}}^{*}$

Fig. 1 (continued) 


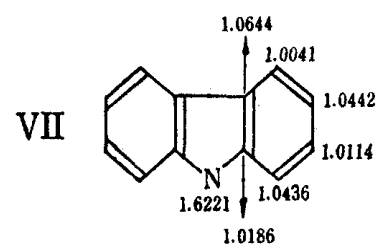

$\mathrm{qr}$<smiles>NC(=O)c1ccccc1O</smiles>

qr

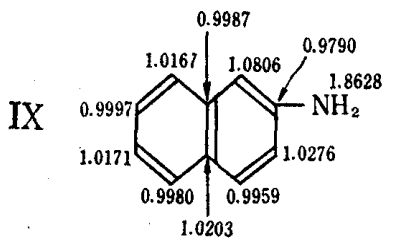

qr<smiles>[X]c1ccccc1Cl</smiles>

$q \mathbf{r}$

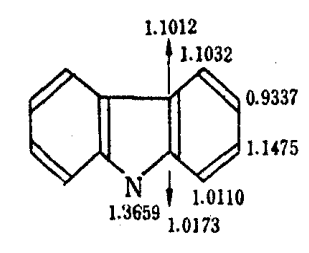

$q \stackrel{*}{r}$

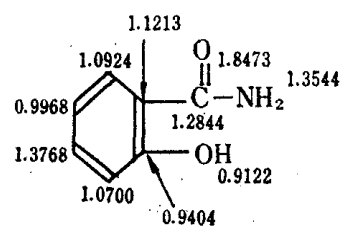

$\mathrm{q}$ *

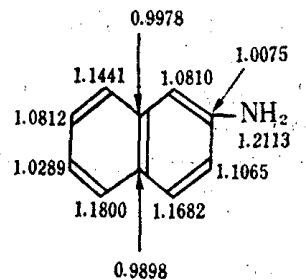

$q \stackrel{*}{r}$<smiles>Clc1ccccc1Cl</smiles>

$q \stackrel{*}{r}$

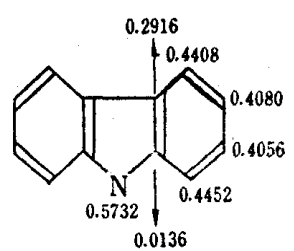

Fr

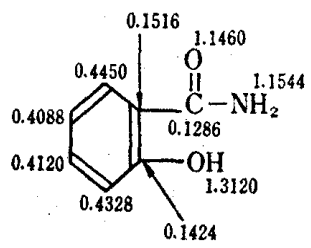

$\mathrm{Fr}$

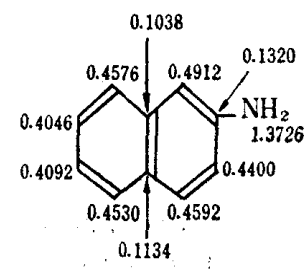

Fr<smiles>Clc1ccccc1</smiles>

Fr

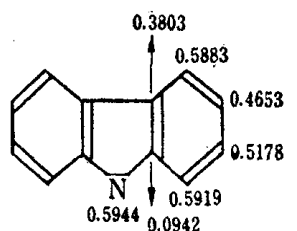

Fr<smiles>CC1(C(N)=O)C2CC3CC(C2)CC1(O)C3</smiles>

$\mathrm{Fr}_{\mathrm{r}}^{*}$<smiles>Cc1cccc(C)c1N</smiles>

$\mathrm{Fr}_{\mathrm{r}}^{*}$<smiles>Clc1ccccc1</smiles>

$\mathrm{Fr}_{\mathrm{r}}^{*}$

Fig. 1. Various Reactivity Indices

qr: $\pi$-electron density in the ground state Fr: free valence number in the ground state $\mathrm{qr}^{*}: \pi$-electron density in the first excited state Fr*: free valence number in the first excited state

Subsequently, relationship between the hydroxylated position of these aromatic substances $^{3-13)}$ and the $\pi$-electron density or the free valence number in the case of ground and first electronic excited state was investigated. From the results summarized in Table I, it should be noted that the mechanism of hydroxylation reaction of these aromatic substances by monooxygenase cannot be considered as the nucleophilic reaction in the ground state, electrophilic

3) D.A. Richert, J. Biol. Chem., 189, 763 (1951); L.A. Schukina, Nature, 154, 513 (1944).

4) R.T. Williams, J. Med. Pharm. Chem., 3, 25 (1961).

5) R.C. Longland, Nature, 221, 664 (1969).

6) D.V. Parke, Biochem. J., 44, 242 (1949).

7) R.T. Williams, "Detoxication Mechanisms," Chapman \& Hall, London, 1959. p. 561,

8) R.T. Williams, Biochem. J., 60, 284 (1955).

9) S. Stolman, Toxicology, 1, $100(1960)$.

10) S.E. Wright, Experientia, 18, 416 (1962).

11) R.T. Williams, "Detoxication Mechanisms," Chapman \& Hall, London, 1959. p. 359,

12) E. Boyland, Biochem. J., 69, 601 (1958).

13) J.N. Smith, Biochem. J., 47, $284(1950)$. 
reaction in the first electronic excited state, or the radical reaction either in the ground state or in the first electronic excited state. Further, electrophilic reaction in the ground state also cannot explain the results of these hydroxylation reactions completely. On the other hand, nucleophilic reaction in the first electronic excited state can explain these results admirably.

TABLE I. Relationship between Hydroxylated Position of Aromatic Compounds and Reactivity Indices $a, b)$

\begin{tabular}{|c|c|c|c|c|c|c|c|c|c|}
\hline \multirow{2}{*}{ No. } & \multirow{2}{*}{ Compound } & \multicolumn{2}{|c|}{ Hydroxylated compound } & \multicolumn{3}{|c|}{ Ground state } & \multicolumn{3}{|c|}{$\begin{array}{c}\text { First excited } \\
\text { state }\end{array}$} \\
\hline & & Main produ & By-product & $\mathrm{E}$ & $N$ & $\mathrm{R}$ & $\mathrm{E}$ & $\mathrm{N}$ & $\mathrm{R}$ \\
\hline$I$ & Menadione & phthiocol (rat, rabbit) ${ }^{3)}$ & $\longrightarrow$ & $x$ & $x$ & 0 & $x$ & 0 & 0 \\
\hline II & Coumarin & $\begin{array}{l}\text { 3-hydroxycoumarin(rabbit) } \\
\text { 7-hydroxycoumarin (man) }\end{array}$ & $\begin{array}{l}\text { 7-hydroxy coumarin } \\
\text { (rabbit) }\end{array}$ & $x$ & $x$ & $\mathrm{O}$ & $x$ & O & $x$ \\
\hline III & Aniline & $p$-aminophenol (rabbit) $\left.{ }^{6}\right)$ & o-aminophenol (rabbit) ${ }^{6}$ ) & 0 & $x$ & $x$ & $x$ & 0 & $x$ \\
\hline $\mathbb{N}$ & Pyridine & 3-hydroxypyridine (rabbit) ${ }^{7}$ ) & - & $\mathrm{O}$ & $x$ & $x$ & $x$ & $\bigcirc$ & $x$ \\
\hline $\mathrm{V}$ & Quinoline & 3-hydroxyquinoline(rabbit) ${ }^{8}$ & $\begin{array}{l}\text { 6-hydroxyquinoline } \\
\text { (rabbit) } \\
\text { 8-hydroxyquinoline } \\
{\text { (rabbit })^{8)}}^{8}\end{array}$ & $\mathrm{O}$ & $x$ & $x$ & $x$ & 0 & $x$ \\
\hline $\mathrm{M}$ & Salicylic acid & gentisic acid $(\operatorname{man})^{9)}$ & $\begin{array}{l}\text { 2,3-dioxybenzoic acid } \\
(\text { man })^{9)} \\
\text { 2,3,5-trioxy benzoic acid } \\
(\text { man })^{9)}\end{array}$ & O & $x$ & $x$ & $x$ & 0 & $x$ \\
\hline VII & Carbazole & 3-oxycarbazole (rabbit, rat) ${ }^{\mathbf{1 0}}$ & - & O & $x$ & $x$ & $x$ & $\mathrm{O}$ & $x$ \\
\hline VIII & Salicylamide & gentisic amide (rat) ${ }^{11)}$ & $\begin{array}{l}\text { gentisic amide }(\operatorname{man})^{11)} \\
\text { 2,3-dioxybenzoic amide } \\
\quad(\operatorname{man})^{11)}\end{array}$ & $x$ & $x$ & $x$ & $x$ & $\bigcirc$ & $x$ \\
\hline $\mathbb{X}$ & $\beta$-Naphthylamine & $\begin{array}{l}\text { 5-hydroxy } \beta \text {-naphthylamine } \\
\text { (rat, rabbit) })^{12)}\end{array}$ & 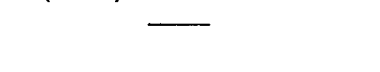 & $x$ & $x$ & $x$ & $x$ & 0 & $x$ \\
\hline $\mathrm{X}$ & Chlorobenzene & $p$-chlorophenol (rabbit) $\left.{ }^{13}\right)$ & & $x$ & $x$ & $x$ & $x$ & O & $x$ \\
\hline
\end{tabular}

a) E: electrophilic reaction; $\mathrm{N}$ : nucleophilic reaction; $\mathrm{R}$ : radical reaction

b) $O$ show the case that the result of hydroxylation reaction can be explained as $E, N$, or $R$, respectively.

$x$ show the case that the result of hydroxylation reaction cannot be explained as $\mathrm{E}, \mathrm{N}$, or $\mathrm{R}$, respectively.

Hence, it may be concluded that some nucleophilic reactions in the first electronic excited state of substrate seem to play an important role in the mechanism of hydroxylation reaction of aromatic substances by monooxygenase, and in this connection, it may be assumed that $\mathrm{O}_{2}^{-14,15)}$ as a nucleophilic reagent seems to participate in these reactions. The present results show that $\pi$-electron density in the first electronic excited state will in future become an indicator for predicting the hydroxylated compounds of some kinds of aromatic substances.

Research Laboratories, Chugai Pharmaceutical Co., Ltd., Takada, Toshima-ku, Tokyo

Received April 25, 1969
KENJI. KUMAKI

Shun-ICHI Hata

Koji Mizuno

SuIICHI TOMIOKA

14) E. Saito and B.H. Bielski, J. Am. Chem. Soc., 83, 4467 (1961).

15) I. Yamazaki, Yuki Gosei Kagaku Kyokai Shi, 27, 53 (1969). 